\title{
Phenotypic and Genetic Analysis of Biofilm Formation by Staphylococcus epidermidis
}

\author{
Iveta Līduma1, 2, 3, Tatjana Tračevska ${ }^{1,2,3}$, Uǵgis Bērs ${ }^{2,3}$, Aija Žileviča ${ }^{1}$ \\ ${ }^{1}$ Faculty of Medicine, University of Latvia, ${ }^{2}$ Faculty of Biology, University of Latvia, \\ ${ }^{3}$ Laboratory of Bioanalytical and Biodosimetry Methods, Latvia
}

\begin{abstract}
Key words: Staphylococcus epidermidis; nosocomial infections; biofilm formation; virulence factors.
\end{abstract}

Summary. Objective. The most important virulence factor of Staphylococcus epidermidis is their capability to form a biofilm on the surfaces of implanted medical devices. The accumulative phase of biofilm formation is linked to the production of intercellular adhesin encoded by the icaADBC operon and accumulation-associated protein by the aap gene.

The aim of the study was to investigate biofilm formation phenotypically and genetically in clinical strains of $S$. epidermidis in comparison with commensal strains.

Material and Methods. The study was carried out in 4 hospitals in Riga, Latvia. In total, 105 clinical strains of Staphylococcus epidermidis isolated from patients' blood $(n=67)$ and intravenous catheters $(n=38)$ in a case of laboratory-confirmed bacteremia were studied. Moreover, 60 Staphylococcus epidermidis commensal strains isolated from nose epithelium of healthy people were included as a control group. Appearance of the icaA and aap genes was tested by polymerase chain reaction. The microtiter plate method was used.

Results. Biofilm formation was detected in 50 (47\%) of Staphylococcus epidermidis isolates in the clinical group and 15 (25\%) of isolates in the control group $(P=0.0049)$. Among 50 biofilmforming clinical isolates, 46 (92\%) were positive for the icaA and/or aap genes. The icaA and aap genes were not found only in 4 strains.

Conclusions. The clinical isolates of Staphylococcus epidermidis were more likely to form biofilms than the commensal strains. The carriage of the icaA or aap gene alone, or their absence, is not applicable as a molecular marker for the discrimination invasive Staphylococcus epidermidis strains from contaminants.

\section{Introduction}

Coagulase-negative staphylococci and, in particular, Staphylococcus epidermidis (S. epidermidis), have emerged as major nosocomial pathogens associated with the infections of implanted medical devices.

S. epidermidis is primarily a normal inhabitant of a healthy human mucosa and skin. As a commensal bacterium, it has a low pathogenic potential, but during the last decades, some strains have transformed into highly adaptable human pathogens (1, 2). Since the early 1980s, these strains have emerged as important nosocomial pathogens and are considered as the ones of the most frequent health care-associated microorganisms, featuring prominently among blood culture isolates and, as mentioned above, especially causing infections associated with implanted devices, such as intravascular catheters and prosthetic implants $(3,4)$.

The virulence factors of coagulase-negative staphylococci, including $S$. epidermidis, have not been well defined yet.

Correspondence to I. Lìduma, "Ezerlici," 2130 Ulbroka, Riga reg., Latvia. E-mail: iveta.liduma@lu.lv
Biofilm formation is thought to be the major pathogenic factor of $S$. epidermidis. A study on the pathogenesis of $S$. epidermidis infections by Peters et al. indicated a key role of biofilm formation in a successful colonization of foreign bodies (5).

The first step in the interaction between microorganisms and a host is adherence to the substrate and then formation of mucoid slime, an extracellular polysaccharide referred to as a biofilm. The initial bacterial adhesion is nonspecific, which is followed by a specific adhesion, mediated by a specific receptor $(6,7)$. Microbial cells are embedded in a slime layer; there they interact among themselves and adhere one to another, thus, forming a tight layer. S. epidermidis biofilm is characterized by a low metabolism of cells, decreased transcription and translation, and a shift from aerobic production of energy to fermentation, resulting in a nonaggressive and protected mode of growth that is less sensitive to antibiotics and the host immune defense $(8,9)$.

Biofilm production is mediated by several factors, mainly by adhesion molecules and a possibility to produce the great amounts of slime (10). Ac- 
cording to the recent studies of adhesion molecules, polysaccharide intercellular adhesin (PIA) is considered to be the most important. PIA is a $\beta-1,6$-linked $\mathrm{N}$-acetylglucosamine, which is responsible for a cell-to-cell attachment $(11,12)$. PIA production in S. epidermidis is encoded by the icaADBC operon.

Other factor that mediates biofilm formation independently of PIA is accumulation-associated protein (AAP) encoded by the aap gene. AAP is essential for biofilm development and is involved in the second accumulation phase of biofilm formation (13). Both icaA and aap genes are used as the potential virulence markers.

Therefore, the leading researchers, such as Vandecasteele et al. (14), have reported the icaADBC operon and the aap gene to be the most important genetic determinants of biofilm production and virulence markers.

One of the major challenges of daily diagnostic practice in a clinical microbiology laboratory is to discriminate between the clinically significant invasive strains of $S$. epidermidis and skin contaminants. Finding the virulence markers allowing a rapid differentiation between virulent and nonvirulent microorganisms would be of great clinical importance.

The aim of this study was to investigate biofilm formation phenotypically and genetically in the clinical strains of $S$. epidermidis in comparison with the commensal strains.

\section{Material and Methods}

Bacterial Strains. In total, 105 clinical strains of $S$. epidermidis isolated from patients' blood $(n=67)$ and intravenous catheters $(\mathrm{n}=38)$ in a case of bacteremia were studied. The patients' age ranged from 35 to 85 years (mean, 61 years).

The clinical definition of $S$. epidermidis bacteremia was based on the CDC criterion of $\geqslant 2$ positive blood cultures within 48 hours along with the symptoms of infection (i.e., fever $>38^{\circ} \mathrm{C}$, chills, or hypotension) that were not related to another infection. The patients were considered to have true coagulase-negative staphylococcal (CoNS) bacteremia if they met the CDC clinical criteria and if identical species and strains were isolated from positive blood culture sets. The blood cultures were processed by using the BacT/Alert ${ }^{\mathrm{TM}}$ system (Biomerieux, France) for aerobic and anaerobic bacteria.

For the analysis, $38 \mathrm{~S}$. epidermidis isolates were recovered from catheters ( $>1000$ colony-forming units on a quantitative catheter culture) of the patients with suspected bacteremia. Besides, 60 S. epidermidis isolates from normal nose epithelium flora were included in the control group, which was represented by $20-22$-year-old healthy people not involved in public health care.

S. epidermidis cultures, isolated from specimens, were identified on the basis of colony morphology, gram staining, and positive results of catalase and negative coagulase tests (Slidex Staph Plus, bioMérieux) and then identified up to a species level using the $\mathrm{BBL}^{\mathrm{TM}} \mathrm{Crystal}^{\mathrm{TM}}$ identification system (Becton, Dickinson and Company, USA).

The following international reference strains were used as controls: the nonbiofilm producers S. epidermidis ATCC 12228 and the biofilm producers S. epidermidis ATCC 35984 (RP62A).

Tissue Culture Plate Method. The biofilm formation using a microtiter plate was carried out as described by Christensen et al. (15). Overnight-grown bacteria in trypticase soy broth (TSB) were diluted (1:100), and $200-\mathrm{mL}$ portions were inoculated into sterile 96-well flat bottom polystyrene microtiter plates (Sarstedt, Inc, Newton, USA). The incubation was carried out at $36^{\circ} \mathrm{C}$ for $22-24$ hours before the removal of the cultures. The wells were washed 3 times with phosphate-buffered saline (PBS, pH 7.2 ) and then air dried and stained with $0.4 \%$ safranin. The optical density of the wells was measured at $490 \mathrm{~nm}$ using an ELISA microplate reader (Biochrom Asys Expert Plus GmbH, Austria).

An optical density of 0.12 was chosen to distinguish biofilm producers from those that did not form a biofilm. Biofilm-positive and negative strains of $S$. epidermidis were included in each plate, and the medium was also added without bacteria.

Isolation of Genomic DNA. All the isolates were grown overnight in brain-heart infusion broth. The bacteria were pelleted, and the DNA isolation was performed with a genomic DNA purification kit (Fermentas Life Science, EU) according to the manufacturer's instructions. DNA concentration was measured using a NanoDrop 1000 spectrophotometer (NanoDrop Technologies, USA).

Detection of icaA and aap Genes. All the isolates were tested for the icaA and aap genes using the polymerase chain reaction (PCR) method. The primers were synthesized by Invitrogen Life Technologies (UK).

The primer sequences were as follows: icaA 1,5 'CAC GTG CTC TAT GGA TG-3'; icaA2, 5'-CCG TTG GAT ATT GCC TCT GT-3'; aap1, 5'-ATA CAA CTG GTG CAG ATG GTT G-3'; and aap2, 5'-GTA GCC GTC CAA GTT TTA CCA G-3'.

All the PCRs were performed on the GeneAmp PCR System 9700 (PE Applied Biosystems, Foster City, CA, USA). The cycling conditions were as follows: preheating for 5 minutes at $94^{\circ} \mathrm{C}$, followed by 25 cycles of 30 seconds at $94^{\circ} \mathrm{C}, 60$ seconds at $55^{\circ} \mathrm{C}$, and 60 seconds at $72^{\circ} \mathrm{C}$. In each PCR, a positive control (S. epidermidis ATCC 35984, RP62A) and a negative control (distilled water) were included. The amplified products were analyzed by agarose gel electrophoresis. The amplicon of icaA was 502 $\mathrm{bp}$, and that of aap, $399 \mathrm{bp}$. 
Statistical Analysis. The GraphPad Prism program version 5.0 was used. All $P$ values were calculated with the Fisher exact test.

\section{Results}

Using the microtiter plate method for the detection of slime production of $S$. epidermidis strains, the following results were obtained.

Nearly half $(\mathrm{n}=50,47.6 \%)$ of 105 S. epidermidis isolates of the clinical group were biofilm producers. In the other 55 clinical strains, the production of extracellular slime was not registered. In the control group, the number of biofilm-positive strains was lower: only $15(25 \%)$ of the 60 cultures were slime producers ( $P=0.0049$; OR, 2.727; 95\% CI, 1.356-5.486).

Specific molecular characteristics, i.e., the presence of the $i c a A$ and aap genes, were studied separately in the biofilm-producing and nonproducing strains. The results are presented in Table 1.

Among 50 biofilm-forming clinical isolates, 46 $(92 \%)$ were positive for ica $A$ and/or aap genes. Only in 4 strains, the icaA and aap genes were not found. Thus, the association between molecular markers and biofilm production was observed.

The analysis of 55 clinical strains in which the biofilm production was not found revealed the following: $13(23.6 \%)$ of the strains were negative for the ica/aap genes, and 32 were positive for the icaA/ aap, but did not produce an extracellular polymer.

Of the 60 control isolates, $45(75 \%)$ did not form biofilms, and slime production was not observed. In $19(42.2 \%)$ of them, the genetic markers ica/aap were not found, but the other 26 were positive for one or both genes. In 15 biofilm-positive control cultures, $13(86.6 \%)$ were positive for the ica/aap genes, and only 2 isolates were negative.

The main attention was focused on the pathogenic $S$. epidermidis strains. Therefore, the strains isolated from blood and catheters were separately analyzed (Table 2). The results showed that they corresponded to the previously described principles, i.e., about half of strains were capable of forming biofilms, and the main part of them was ica/aap positive. There were $26(38.8 \%)$ biofilm-positive $S$. epidermidis isolates from blood cultures $(n=67)$ and $21(55.2 \%)$ biofilm-positive isolates from catheters $(\mathrm{n}=38)$.

The presence of the aap/icaA genes and biofilm formation was slightly more often observed in catheter-related isolates than blood culture isolates; however, this difference did not reach the statistical significance ( $P=0.152$; OR, 0.513; 95\% CI, 0.229-1.150).

\section{Discussion}

The main virulence factor of coagulase-negative staphylococci is considered to be their ability to form biofilms. The pathogenesis of staphylococcal diseases depends on their capability to adhere and then form a mucoid layer (slime layer), especially in intravenous catheter- or prosthetic device-related bacteremia. The elaboration of a biofilm is a complex process in which different proteins play a role, with adhesion molecules, accumulation-associated proteins, and factors responsible for cell-to-cell interactions among them.

The objective of the present work was to search for $S$. epidermidis virulence factors, applying phenotypic and genotypic methods, and to evaluate

Table 1. Biofilm Formation and Presence or Absence of icaA and aap Genes in Clinical and Commensal S. epidermidis Isolates

\begin{tabular}{lccccc}
\hline \multicolumn{1}{c}{$\begin{array}{c}\text { Biofilm Formation icaA } \\
\text { and aap Genes }\end{array}$} & $\begin{array}{c}\text { Clinical Group } \\
\mathrm{n}=105\end{array}$ & $\begin{array}{c}\text { Control Group } \\
\mathrm{n}=60\end{array}$ & $P$ & Odds Ratio & 95\% Confidence Interval \\
\hline $\mathrm{BF}+/$ aap + /icaA+ & $27(25.7)$ & $5(8.3)$ & 0.0073 & 3.808 & $1.380-10.510$ \\
$\mathrm{BF}+$ /aap-/icaA- & $4(3.8)$ & $2(3.3)$ & 1.0000 & 0.871 & $0.155-4.903$ \\
$\mathrm{BF}+$ /aap+ or icaA+ & $19(18.1)$ & $8(13.3)$ & 0.5148 & 1.436 & $0.587-3.515$ \\
$\mathrm{BF}-/$ aap+/icaA+ & $14(11.4)$ & $8(13.3)$ & 1.000 & 1.000 & $0.393-2.543$ \\
$\mathrm{BF}-/$ aap - /icaA - & $13(12.4)$ & $19(31.7)$ & 0.0039 & 0.305 & $0.138-0.676$ \\
BF-/aap+ or icaA+ & $28(26.7)$ & $18(30)$ & 0.7189 & 0.849 & $0.421-1.711$ \\
\hline
\end{tabular}

Values are number (percentage). BF, biofilm formation using the tissue culture plate method.

Table 2. Biofilm Formation and Presence or Absence of icaA and aap Genes in S. epidermidis Strains Isolated from Blood and Catheters

\begin{tabular}{|c|c|c|c|c|c|}
\hline $\begin{array}{c}\text { Biofilm Formation icaA } \\
\text { and aap Genes }\end{array}$ & $\begin{array}{c}\text { Blood Cultures } \\
n=67\end{array}$ & $\begin{array}{c}\text { Catheters } \\
\mathrm{n}=38\end{array}$ & $P$ & Odds Ratio & $95 \%$ Confidence Interval \\
\hline $\mathrm{BF}+/ a a p+/ i c a A+$ & $10(14.9)$ & $10(26.3)$ & 0.2092 & 0.517 & $0.200-1.336$ \\
\hline $\mathrm{BF}+/ a a p-/ i c a A-$ & $5(7.5)$ & $3(7.9)$ & 1.000 & 0.812 & $0.183-3.586$ \\
\hline $\mathrm{BF}+/$ aap + or $i c a A+$ & $11(16.4)$ & $8(21)$ & 0.6015 & 0.692 & $0.256-1.871$ \\
\hline $\mathrm{BF}-/$ aap $+/$ icaA+ & $8(11.9)$ & $5(13.1)$ & 1.000 & 0.874 & $0.271-2.814$ \\
\hline $\mathrm{BF}-/$ aap-/icaA- & $8(11.9)$ & $3(7.9)$ & 0.5401 & 1.741 & $0.450-6.742$ \\
\hline $\mathrm{BF}-/$ aap + or $i c a A+$ & $25(37.3)$ & $9(23.7)$ & 0.1944 & 1.918 & $0.782-4.704$ \\
\hline
\end{tabular}

Values are number (percentage). BF, biofilm formation using the tissue culture plate method. 
the studied features as the possible criteria for the discrimination between the virulent clinical nosocomial and nonvirulent contaminant strains. The attention was focused mainly on the capability of S. epidermidis to produce biofilms and on the association between biofilm production and the genetic markers, icaA and aap genes.

Our data revealed that biofilm formation was more characteristic of the clinical strains of $S$. epidermidis. The difference comparing with the control strains was statistically significant. The present study showed that the percentage of the biofilmproducing strains was $47.6 \%$. It is very close to the one found by Arciola et al. (16), i.e., 46\%; in other studies, a higher percentage of biofilm-producing cells were documented Ziebuhr et al. (17) reported that $87 \%$ of clinical S. epidermidis isolates were biofilm producers. At the same time, other phenotypic study by Rohde et al. (18) showed contrary results: the authors failed to find an association between the clinical significance of $S$. epidermidis and the ability to form a biofilm in vitro.

In the present study, the association between biofilm formation and presence of the icaA and aap genes was positive: $92 \%$ of the clinical biofilm-producing strains and $86.6 \%$ of the commensal strains were positive for the icaA/aap genes.

Similar data were obtained by Eftekhar and Mirmohamadi (19), who concluded that $S$. epidermidis isolated from patients with symptomatic infections were more virulent, and the expression level of $i c a A D B C$ and other regulatory factors independent

\section{References}

1. Ziebuhr W. Staphylococcus aureus and Staphylococcus epidermidis: emerging pathogens in nosocomial infections. Contrib Microbiol 2001;8:102-7.

2. Zilevica A, Tracevska T, Liduma I, Viesturs U. Discrimination between contaminating strains of Staphylococcus epidermidis. Int J Bioautomation 2010;14:179-86.

3. Miragaia M, Couto I, Pereira SFF, Kristinson KG, Westh H, Jarløv JO, et al. Molecular characterization of methicillin-resistant Staphylococcus epidermidis clones: evidence of geographic dissemination. J Clin Microbiol 2002;40:2:430-8.

4. Mack D, Rohde H, Harris LG, Davies AP, Horstkotte MA, Knobloch J. Biofilm formation in medical device-related infection. Int J Artif Organs 2006;29:343-59.

5. Peters G, Locci R, Pulverer G. Adherence and growth of coagulase-negative staphylococci on surfaces of intravenozus catheters. J Infect Dis 1982;146:479-82.

6. O'Gara JP, Humphreys H. Staphylococcus epidermidis biofilms: importance and implications. J Med Microbiol 2001;50:582-7.

7. Rohde H, Burdelski C, Bartscht K, Hussain M, Buck F, Horstkotte MA. Induction of Staphylococcus epidermidis biofilm formation via proteolytic processing of the accumulation-associated protein by staphylococcal and host proteases. Mol Microbiol 2005;55:1883-95.

8. McCann MT, Gilmore BF, Gorman SP. Staphylococcus epidermidis device related infections: pathogenesis and clini- of PIA synthesis was of importance.

In some ica/aap-positive strains, biofilm production was not observed; this can be explained by an extreme sensitivity of this feature to different environmental factors like antibiotic concentration in a patient's body, high temperature, glucose level, etc. Our findings are in line with those of Frebourg et al. (20).

In summary, we propose that in biofilm formation by coagulase-negative staphylococci, the ica operon and the aap gene play a crucial role, but they are not the only players in the big orchestra as the regulation of biofilm development is a complex process. Detection of biofilm-producing icaA/aapnegative strains indicates this.

\section{Conclusions}

The clinical isolates of Staphylococcus epidermidis were more likely to form biofilms than the commensal strains. The carriage of the icaA or aap gene alone, or their absence, is not applicable as a molecular marker for the discrimination invasive Staphylococcus epidermidis strains from contaminants.

\section{Acknowledgments}

The study was funded by the European Social Fund project "Interdisciplinary studies in biosafety; capacity fortification" (No. 2009/0224/1DP/ 1.1.1.2.0/09/APIA/VIAA/0).

\section{Statement of Conflict of Interest}

The authors state no conflict of interest.

cal management. J Pharm Pharmacol 2008;60(12):1551-71.

9. Fitzpatrick F, Humphreys H, O'Gara JP. The genetics of staphylococcal biofilm formation. Clin Microbiol Infect 2005;11:967-73.

10. Kong KF, Vuong C, Otto M. Staphylococcus quorum sensing in biofilm formation and infections. Int J Med Microbiol 2006;296(2-3):133-9.

11. Stevens NT, Tharmabala M, Dillane T, Greene CM, O'Gara JP. Biofilm and the role of the ica operon and aap in Staphylococcus epidermidis isolates causing neurosurgical meningitis. J Clin Microb Inf Dis 2008;14:716-30.

12. Heilmann C, Schweitzer O, Gerke C, Vanittanakom N, Mack D, Gotz F. Molecular basis of intercellular adhesion in the biofilm-forming Staphylococcus epidermidis. Mol Microbiol 2004;20:1083-91.

13. Ninin E, Caroff N, Espaze E, Maraillac J, Lepelletier D, Milpied N, et al. Assessment of ica operon carriage and biofilm production in Staphylococcus epidermidis isolates causing bacteraemia in bone marrow transplant recipients. Clin Microbiol Infect 2006;12:446-52.

14. Vandercasteele SI, Peetermans WL, Merckx R, Rijnders BJA, van Eldere J. Reliability of the ica, aap and atlE genes in the discrimination between invasive, colonizing and contaminant Staphylococcus epidermidis isolates in the diagnosis of catheter-related infections. J Clin Microbiol 2003; 9:114-9.

15. Christensen GD, Baddour L, SimpsonWA. Phenotypic var- 
iation of Staphylococcus epidermidis slime production in vitro and in vivo. Infect Immun 1987;55:2870-7.

16. Arciola CR, Baldassarri L, Montanaro L. Presence of icaA and icaD genes and slime production in a collection of staphylococcal strains from catheter-associated infections. J Clin Microbiol 2001;39(6):2151-6.

17. Ziebuhr W, Heilmann C, Gotz F, Meyer P, Wilms K, Straube E, et al. Detection of the intercellular adhesion gene cluster (ica) and phase variation in Staphylococcus epidermidis blood culture strains and mucosal isolates. Infect Immun 1997;65:890-6.

18. Rohde H, Mack D, Christner M, Burdelski C, Franke G,
Knobloch JK. Pathogenesis of staphylococcal device related infections: from basic science to new diagnostic, therapeutic and prophylactic approaches. Rev Med Microbiol 2006; $17: 45-54$

19. Eftekhar F, Mirmohamadi Z. Evaluation of biofilm production by Staphylococcus epidermidis isolates from nosocomial infections and skin of healthy volunteers. Int J Medicine Medical Scie 2009;10:438-41.

20. Frebourg NB, Lefevre S, Baert S, Lemeland JF. PCR-based assay for discrimination between invasive and contaminating Staphylococcus epidermidis strains. J Clin Microbiol 2000;38:877-80.

Received 29 September 2011, accepted 23 June 2012 